\title{
Clinical consequences of the presence of anti-RNA Pol III antibodies in systemic sclerosis
}

\author{
Ewa Wielosz, Magdalena Dryglewska, Maria Majdan \\ Chair and Department of Rheumatology and Connective Tissue Diseases, Medical University of Lublin, Lublin, Poland \\ Adv Dermatol Allergol 2020; XXXVII (6): 909-914 \\ DOI: https://doi.org/10.5114/ada.2020.102107
}

\begin{abstract}
Introduction: Anti-RNA polymerase III (a-RNA Pol III) antibodies are marker antibodies in patients with systemic sclerosis (SSc).

Aim: To assess the prevalence of a-RNA Pol III in patients with SSc and to identify the differences in the disease picture in SSc patients with and without a-RNA Pol III antibodies.

Material and methods: The study was performed in 126 SSc patients. The subtype of SSc, incidence of internal organ involvement, malignancy, death and serological profiles were determined in the entire group. The study groups were studied according to the presence of antibodies by applying the commercial test - EUROLINE SSc Profile. Due to the presence of a-RNA Pol III, patients were divided into two groups: the a-RNA Pol III (+) SSc group of 19 patients and the a-RNA Pol III (-) SSc group of 107 patients.

Results: A-RNA Pol III were present in 19/126 patients with SSc (15\%), 13/19 (68.4\%) patients had no other SSc marker antibodies. A-RNA Pol III were more common in patients with diffuse cutaneous SSc $(p=0.049)$. We showed a significant positive association between a-RNA Pol III and occurrence of malignancy $(p=0.007)$, scleroderma renal crisis ( $p=0.001)$ and decreased DLCO $(p=0.007)$.

Conclusions: Anti-a-RNA Pol III antibodies are common in patients with SSc, particularly with a diffuse subtype. In more than $50 \%$ of patients with a-RNA Pol III antibodies, they may be present as the sole marker of antibodies. In SSc, a-RNA Pol III antibodies are frequently associated with malignancy occurrence, kidney and lung involvement.
\end{abstract}

Key words: systemic sclerosis, anti-RNA Pol III antibodies, malignancies.

\section{Introduction}

Systemic sclerosis (SSc) is a connective tissue disease characterized by microangiopathy, fibrosis and immune dysregulation. Autoimmunity is mainly considered to be involved in the aetiology and pathogenesis of SSc. Antinuclear antibodies (ANAs) detected by indirect immunofluorescence (IIF) technique are present in 95-99\% of patients with SSc [1]. It is known that a specific serological profile is associated with clinical manifestations and prognosis in SSc. Anti-topoisomerase I (anti-topo I), anti-centromere autoantibodies (ACAs) and anti-RNA polymerase III (a-RNA Pol III) antibodies are marker antibodies which are detected in sera of patients with SSc. Furthermore, they are included in the classification criteria of SSc and associated with a typical subset of disease and prognosis [2]. Sera positive for a-RNA Pol III antibodies produce a speckled staining pattern IIF, but also nucleolar staining if a-RNA Pol I antibodies coexist [3].
According to data, a-RNA Pol III antibodies are found in approximately $4-25 \%$ of patients with SSc, particularly with a diffuse subtype of disease [4, 5]. According to the literature, a-RNA Pol III antibodies are strongly associated with severe clinical manifestations of the disease such as scleroderma renal crisis (SRC), heart involvement, gastric antral vascular ectasia as well as higher rates of SSc-related mortality [6, 7]. Additionally, there are many data demonstrating that a-RNA Pol III antibodies are associated with an increased risk of malignancy in patients with SSc [8-10].

\section{Aim}

The aim of the present study was assess the prevalence of a-RNA Pol III antibodies in patients with SSc and to identify the differences in the disease picture in patients with the presence of a-RNA Pol III.

Address for correspondence: Ewa Wielosz MD, PhD, Chair and Department of Rheumatology and Connective Tissue Diseases, Medical University of Lublin, 8 Jaczewskiego St, 20-090 Lublin, Poland, phone: +48 81724 47 90, fax: +48 8172445 15, e-mail: ewa.wielosz@wp.pl Received: 7.02.2019, accepted: 26.04.2019. 


\section{Material and methods}

The study included 126 European Caucasian SSc patients hospitalized consecutively in the Department of Rheumatology and Connective Tissue Diseases, Medical University of Lublin. All patients gave written informed consent to participate in the study according to the Helsinki Declaration of 1975, as revised in 2000. The study design was approved by the ethical committee. All patients fulfilled the American College of Rheumatology (ACR)/European League against Rheumatism (EULR) classification criteria of SSc [2]. The subtype of SSc, incidence of internal organ involvement, the prevalence of malignancy, death and serological profiles were determined in the entire group. According to Le Roy et al., patients were classified as limited cutaneous (Ic) SSc or diffuse cutaneous (dc) SSc [11] (Table 1). Organ involvement was assessed according to the clinical symptoms and the results of diagnostic tests. Interstitial lung disease (ILD) was defined as "a ground glass" pattern or bibasilar pulmonary fibrosis revealed on a high-resolution computer tomography (HRCT) scan. The DLCO test (\% of predicted diffusing capacity for carbon monoxide) and total lung capacity (TLC) testing were performed to assess the pulmonary function. Heart involvement was established as arrhythmia, conduction disturbances or heart failure. Pulmonary arterial hypertension (PAH) was defined as systolic pulmonary arterial pressure (SPAP) > $35 \mathrm{~mm} \mathrm{Hg}$ in Doppler echocardiography and was determined only at rest. Myalgia or myositis was defined as pain or weakness of muscles and/or increased serum creatine phosphokinase (CPK) concentration. Joint involvement was determined as joint tenderness and swelling. Gastrointestinal tract involvement was defined according to clinical symptoms such as dysphagia, heartburn, diarrhoea or bloating and was examined by a barium swallow. Renal involvement was defined as the development of scleroderma renal crisis (SRC) or presence of proteinuria and elevated serum creatinine concentration. Calcinosis and digital erosions were also assessed. Serum samples were obtained from each patient. Moreover, the presence of

Table 1. Characteristics of the study group

\begin{tabular}{lc}
\hline Parameter & Value \\
\hline Number of patients: & 126 \\
\hline Female & 98 \\
\hline Male & 28 \\
\hline Type of SSc: & 60 \\
\hline dcSSc & 66 \\
\hline IcSSc & $53.6(18.0-81.0)$ \\
\hline Age [years] & $6.3(0.0-23.0)$ \\
\hline Duration of disease [years]
\end{tabular}

dcSSC - diffuse cutaneous systemic sclerosis, IcSSc - limited cutaneous sys temic sclerosis. antibodies was assessed in the study groups, by applying the commercial test - EUROLINE Systemic Sclerosis Profile, which is used to determine antibodies directed against SSc-specific antigens, such as anti-topoisomerase I (anti-Scl-70) and anti-centromere autoantibodies (ACAs), a-RNA Pol III - subunit RP11 and RP 155, as well as more rare anti-PM/Scl, anti-Ku, anti-Th/To anti-Ro52 and autoantibodies against the nucleolus-organizing region-90 (anti-NOR90). Sera were analysed at a dilution of $1: 101$, and autoantibodies were detected using alkaline phosphatase-labelled antihuman IgG. Detection and interpretation of results was carried out electronically using the specific program Euroimmun - EUROLINE Scan (EUROIMMUN AG, Lubeck, Germany). According to the presence of a-RNA Pol III antibodies, patients were divided into two groups -a-RNA Pol III positive group (+) SSc of 19 patients (pts) and a-RNA Pol III negative group (-) SSc of 107 patients.

\section{Statistical analysis}

All calculations were performed with Statistica v. 10.0 software (StatSoft, Krakow, Poland). Data were analysed using the non-parametric $\chi^{2}$ test for comparisons between the groups. $P$-value $<0.05$ was considered as statistically significant.

\section{Results}

According to our data, a-RNA Pol III antibodies were present in 19/126 patients with SSc (15\%), including aRNA Pol III 11 present in 16 patients and a-RNA Pol III 155 in 14 patients. In this group of 13 (68.4\%) patients had no other SSc marker antibodies, such as anticentromere or anti-topoisomerase I antibodies. Moreover, dcSSc was observed more commonly in the a-RNA Pol III positive group than in the a-RNA Pol III negative group $(p=0.049)$. Furthermore, a-RNA Pol III antibodies were more common in patients with dcSSc, compared to lcSSc patients. The clinical characteristics of SSc patients in the a-RNA Pol III positive and a-RNA Pol III negative groups were presented in Table 2. We also showed that a decrease in DLCO was significantly larger in the a-RNA Pol III positive group compared to the a-RNA Pol III negative group $13 / 19$ vs. 38/107 ( $p=0.007$ ) (Figure 1). Moreover, the incidence of SRC was significantly higher in the aRNA Pol III positive group compared to the a-RNA Pol III negative group ( $p=0.001$ ) (Figure 2$)$. In addition, we found a significant positive association between a-RNA Pol III antibodies and occurrence of malignancy 5/19 vs. $7 / 107$ ( $p=0.007$ ) (Figure 3). In the RNA Pol III positive group 3 patients had lung cancer, 1 ovarian cancer and 1 cervical cancer, while there were 2 lung cancers, 1 stomach cancer, 1 breast cancer, 1 kidney cancer, 1 adrenal tumour and 1 brain tumour in the RNA Pol III negative group. The time from onset of Raynaud phenomenon to diagnosis did not differ between the groups. Further- 
Table 2. Clinical characteristics of patients of the SSc a-RNA Pol III (+) and SSc a-RNA Pol III (-) groups

\begin{tabular}{lccc}
\hline Parameter & SSc a-RNA Pol III (+) & SSc a-RNA Pol III (-) & $P$-value \\
\hline \begin{tabular}{l} 
Number of patients: \\
\hline dcSSc
\end{tabular} & 19 & 107 & 0.049 \\
\hline IcSSc & $6(68.4 \%)$ & $47(43.9 \%)$ & \\
\hline \begin{tabular}{l} 
Gender: \\
\hline Female
\end{tabular} & $12(63.2 \%)$ & $60(56.1 \%)$ & NS \\
\hline Male & $7(36.8 \%)$ & $21(19.6 \%)$ & NS \\
\hline Age [years] & $55.11 \pm 9.56(38.0-75.0)$ & $53.57 \pm 14.12(19-81)$ & 0.056 \\
\hline Duration of disease [years] & $3.21 \pm 3.12(0.0-13.0)$ & $5.85 \pm 5.81(0.0-23.0)$ & NS \\
\hline $\begin{array}{l}\text { The time from onset of Raynaud phenomenon to diagnosis } \\
\text { [years] }\end{array}$ & $4.64 \pm 8.02(0.0-30.0)$ & $7.03 \pm 12.4(0.3-30.0)$ & \\
\hline
\end{tabular}

Data were presented as number and percentage. P-value of $<0.05$ was considered statistically significant. dcSSc - diffuse cutaneous systemic sclerosis, IcSSC - limited cutaneous systemic sclerosis.

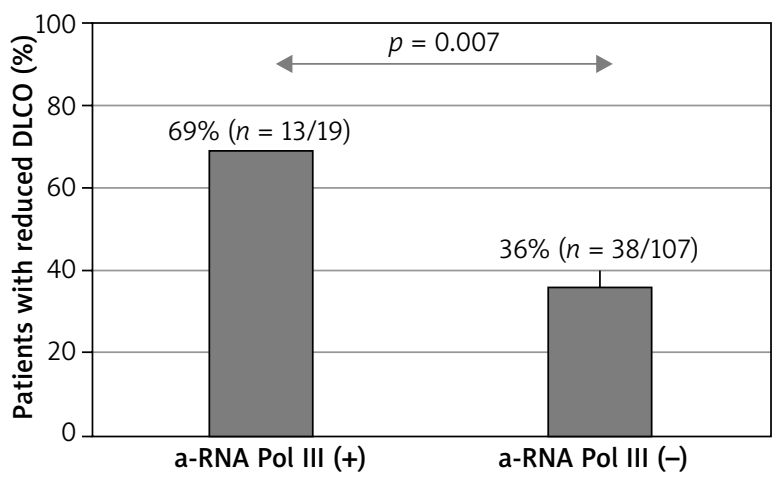

a-RNA Pol III (-) - negative, a-RNA Pol III (+) - positive, DLCO diffusing capacity for carbon monoxide, $n$-number of patients.

Figure 1. Decreased DLCO in a-RNA Pol III (+) and (-) groups

more, there were no significant intergroup differences in ILD, TLC, PAH, heart involvement, gastrointestinal tract involvement, prevalence of arthritis or arthralgia, myalgia, digital ulcerations, contractures, calcinosis, overlap syndromes or mortality (Table 3). Furthermore, we did not find any significant differences in presence of another antibodies between the RNA Pol III positive and negative group (Table 4).

\section{Discussion}

In the present study, we investigated the prevalence of marker antibodies in SSc, such as a-RNA Pol III antibodies. We also tried to assess their usefulness and association with clinical manifestations in patients with SSc. According to our observations, a-RNA Pol III antibodies were found in 15\% of SSc patients. Our findings are similar to those reported in the literature. In the Australian Systemic Sclerosis Cohort Study, Petterson et al. presented that in the SSc group, 81 of 505 patients (16\%) were a-RNA Pol III positive. Moreover, the prevalence of other SSc marker an-

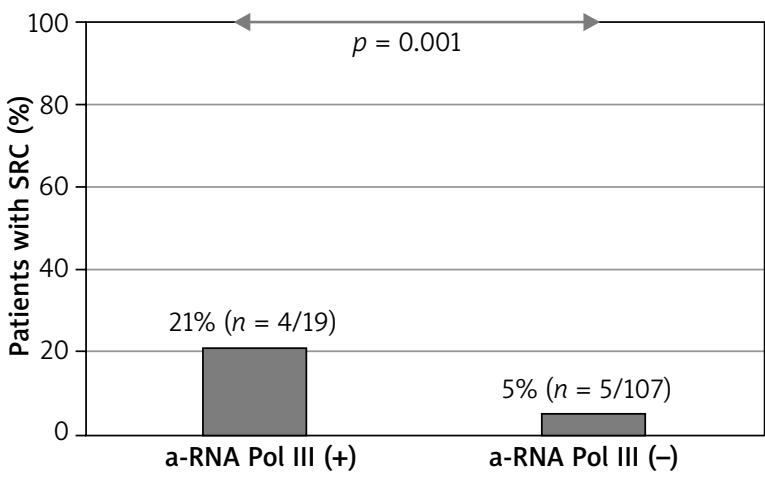

a-RNA Pol III (-) - negative, a-RNA Pol III (+) - positive, $S R C$ - scleroderma renal crisis, $n$ - number of patients.

Figure 2. The prevalence of scleroderma renal crisis in a-RNA Pol III (+) and (-) groups

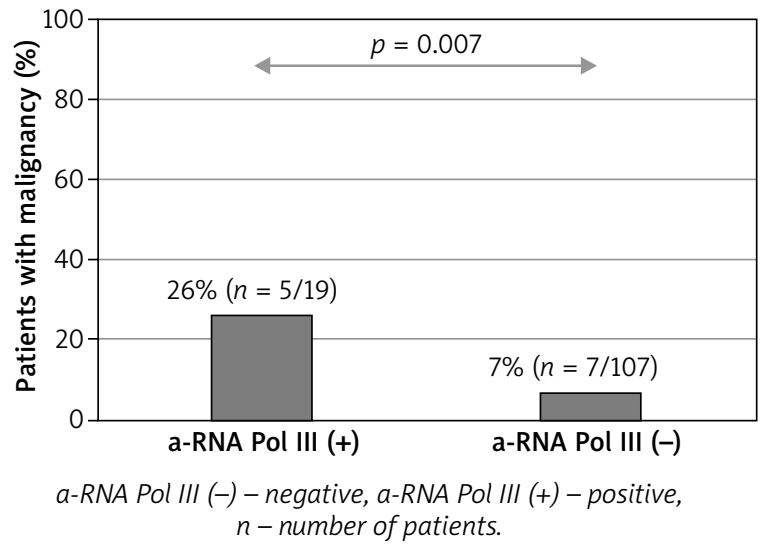

Figure 3. The prevalence of malignancy in a-RNA Pol III (+) and $(-)$ groups

tibodies such as anti-topoisomerase I or anticentromere antibodies in the RNA Pol III positive group was rare [12]. In another study, Mierau et al. from the German Network for Systemic Scleroderma Registry used a commercial line 
Table 3. Comparison of selected clinical parameters in a-RNA Pol III (+) and a-RNA Pol III (-) groups of patients with systemic sclerosis

\begin{tabular}{lccc}
\hline Variable & SSc a-RNA Pol III $(+) n=19$ & SSc a-RNA Pol III(-) $n=107$ & $P$-value \\
\hline ILD (HRCT) & $11(57.9 \%)$ & $57(53.3 \%)$ & NS \\
\hline Decreased DLCO & $13(68.4 \%)$ & $38(35.5 \%)$ & 0.007 \\
\hline TLC & $7(36.8 \%)$ & $30(28.0 \%)$ & NS \\
\hline Heart involvement & $6(31.6 \%)$ & $44(41.1 \%)$ & NS \\
\hline PAH (ECHO) & $2(10.5 \%)$ & $26(24.3 \%)$ & NS \\
\hline Myositis & $3(15.8 \%)$ & $19(17.8 \%)$ & NS \\
\hline Arthralgia & $14(73.7 .8 \%)$ & $93(86.9 \%)$ & NS \\
\hline Arthritis & $4(21.1 \%)$ & $37(34.6 \%)$ & NS \\
\hline Gastrointestinal tract involvement & $11(57.9 \%)$ & $68(63.5 \%)$ & NS \\
\hline Calcinosis & $2(10.5 \%)$ & $22(20.6 \%)$ & NS \\
\hline Digital ulcerations & $3(15.8 \%)$ & $23(21.5 \%)$ & NS \\
\hline Death & $5(26.3 \%)$ & $17(15.9 \%)$ & NS \\
\hline Overlap syndrome & $2(10.5 \%)$ & $25(23.4 \%)$ & NS \\
\hline Contractures & $7(36.8 \%)$ & $33(30.8 \%)$ & NS \\
\hline Datawepresented as & & \\
\hline
\end{tabular}

Data were presented as number and percentage. P-value of $<0.05$ was considered statistically significant. a-RNA Pol III positive - (+), a-RNA Pol III negative - (-), ILD - interstitial lung disease, HRCT - high-resolution computer tomography, DLCO - \% of predicted diffusing capacity for carbon monoxide, TLC - total lung capacity, PAH - pulmonary arterial hypertension, ECHO - echocardiography.

Table 4. Comparison of selected serological parameters in a-RNA Pol III (+) and a-RNA Pol III (-) groups of patients with systemic sclerosis

\begin{tabular}{lccc}
\hline Variable & SSc a-RNA Pol III $(+) n=19$ & SSc a-RNA Pol III (-) $n=107$ & $P$-value \\
\hline Anti-Scl 70 & $5(26.3 \%)$ & $43(40.23 \%)$ & NS \\
\hline ACAs & $1(68.4 \%)$ & $25(23.4 \%)$ & NS \\
\hline Anti-fibrillarin & $0(0 \%)$ & $3(2.8 \%)$ & NS \\
\hline Anti-NOR90 & $1(5.3 \%)$ & $5(4.7 \%)$ & NS \\
\hline Anti-Th/To & $0(0 \%)$ & $2(1.9 \%)$ & NS \\
\hline Anti-Pm/Scl & $3(15.9 \%)$ & $18(16.8 \%)$ & NS \\
\hline Anti-Ku & $0(0 \%)$ & $4(3.7 \%)$ & NS \\
\hline Anti-PDGFR & $0(0 \%)$ & $0(0 \%)$ & NS \\
\hline Anti-Ro52 & $6(31.6 \%)$ & $28(26.2 \%)$ & NS \\
\hline
\end{tabular}

Data were presented as number and percentage. P-value of $<0.05$ was considered statistically significant. a-RNA Pol III positive-(+), a-RNA Pol III negative - (-), ACAs - anti-centromere autoantibodies, anti-NOR90 - autoantibodies against the nucleolus-organizing region-90.

immunoassay (Euroimmun) and found that co-expression of any combination of anticentromere, a-RNA Pol III, and topoisomerase I antibodies was rare, while co-expression of additional nonspecific autoantibodies was common $[13,14]$. In our study, we also detected that approximately $70 \%$ of patients with a-RNA Pol III antibodies had no other SSc marker antibodies as anticentromere or anti-topoisomerase I antibodies. In the RNA Pol III group only 1 patient had anticentromere antibodies and 5 had anti-Scl70 antibodies. We did not find coexistence of antiKu or anti-Th/To antibodies with a-RNA Pol III antibodies because of a small number of patients with this antibodies in our study group. The prevalence of antibodies definitely depends on the method of detection. Peterson and Mierau used the same test to assess antibodies as the one in our study, i.e. the EUROLINE Systemic Sclerosis Profile [12, 13]. On the other hand, Satoh et al. assessed the presence and clinical usefulness of a-RNA Pol III antibody measurements by enzyme-linked immunosorbent assay (ELISA) [15]. Furthermore, in Satoh's study, the prevalence of a-RNA Pol III antibodies in selected serum samples was confirmed by another method for the determination of these antibodies - immunoprecipitation (IP) assay. Using ELISA, they found a-RNA Pol III antibodies in $28 / 355$ (11\%) of SSC patients. In addition, they confirmed in the study that the analytical specificity of ELISA was $>98.8 \%$ but its sensitivity was lower compared with the IP assay [15]. Apart from the method of detection of an- 
tibodies, the frequency of a-RNA Pol III antibodies in SSC patients varies among ethnic groups: a higher frequency was observed in North American Caucasian and UK patients (20-25\%) in comparison with French, Chinese or Japanese patients (2-5\%) [3, 4, 7, 8, 16-18]. The next important step in assessment of marker antibodies for SSc, such as a-RNA Pol III antibodies was to identify differences in the clinical picture of the disease and to find the association between clinical manifestations of SSc and the presence of a-RNA Pol III antibodies. According to the literature and our observations, patients with a-RNA Pol III antibodies have dcSSc with rapidly progressive skin thickening and the highest Rodnan Skin Score [19]. Nevertheless, many patients experience rapid regression of skin thickening over time even without treatment [3]. In our study, a-RNA Pol III antibodies were more common in patients with dcSSc (13/19) as compared to IcSSc (6/19). Satoh et al. have reported similar results; in the group of 38 patients with positive a-RNA Pol III antibodies, 32 patients had dcSSc and 6 patients had IcSSc [15]. It is difficult to explain the regression of skin thickening over time without treatment. Some data report that the high titres of a-RNA Pol III antibodies were observed in the early stages of SSc and it could be associated with rapid skin progression at the onset of disease [12]. Analysing the other clinical presentations of the disease, we found a positive association between a-RNA Pol III antibodies and SRC, occurrence of malignancy and decreased DLCO. The literature data are similar to our results $[19,20]$. Satoh et al. have demonstrated that the clinical features associated with positivity for a-RNA Pol III antibodies include a high total skin score and a trend towards the high prevalence of renal crisis [15]. Moreover, Gordon et al. have revealed that on SSc diagnosis the presence of a-RNA Pol III antibodies was associated with future development of SRC [20]. According to Kuwana et al., patients with a-RNA Pol III antibodies have the highest risk of developing SRC, but they rarely develop severe ILD [3]. In Caucasians, about $60 \%$ of patients with SRC had a- RNA Pol III antibodies. The a-RNA Pol III antibodies could be detected in patients with acute renal failure in the course of SRC in the absence of sclerotic skin disease, but these patients eventually develop rapidly progressive skin thickening [3]. Numerous studies have demonstrated that aRNA Pol III positive patients with SSc have a high risk of concomitant malignancy [8-10, 21, 22]. One large study, which used the data from the EULAR scleroderma trials and research (EUSTAR) cohort, revealed that a-RNA Pol III antibodies were associated with gastric antral vascular ectasia, rapid progression of skin involvement, and malignancies concomitant to SSc onset (OR $=7.38,95 \% \mathrm{Cl}$ : 1.61-33.8). When compared with other a-RNA Pol III positive patients, those with concomitant malignancies had older age and more frequent diffuse cutaneous involvement [10]. The same study has suggested that prevalence of cancer in the a-RNA Pol III positive group is similar to that in the a-topo I and anticentromere group but the SSC duration at cancer diagnosis is significantly shorter [3]. In our study we revealed an association between malignancy and presence of a-RNA Pol III antibodies, but the type of malignancy did not differ between the a-RNA Pol III positive and negative group. To sum up, a relationship between cancer and SSc among a-RNA Pol III positive patients suggests that SSC is a para-neoplastic disorder in this subset of patients. On the other hand, there are some controversial data reporting the lack of association between a-RNA Pol III antibodies and malignancy; however, a risk of cancer in SSc was directly associated with the presence of anti-PM/Scl antibodies [23]. In our study, interestingly, a decrease in DLCO was found to be higher in the positive RNA Pol III SSc group compared to the negative group. An explanation of the result is quite difficult. According to the literature, patients with a-RNA Pol III antibody have a lower risk of developing severe ILD [3]. In our results, we took into consideration the fact that 3/13 RNA Pol III positive patients with decreased DLCO had also a-topo I antibodies which are strongly associated with ILD. The literature data indicate that patients with a-RNA Pol III antibodies had an increased risk of gastric antral vascular ectasia, but a lower risk of oesophageal dysmotility; moreover, age at the onset is older and the proportion of males particularly with sclerosing tenosynovitis tends to be higher in patients with a-RNA Pol III antibodies [3, 12, 19, 24]. In the present study, we did not find such correlations.

\section{Conclusions}

According to our results, a-RNA Pol III antibodies are quite common in patients with systemic sclerosis, particularly with a diffuse subtype. In more than $60 \%$ of patients a-RNA Pol III antibodies may be present as the sole marker of SSc antibodies. In SSC a-RNA Pol III antibodies are frequently associated with malignancy occurrence and development of scleroderma renal crisis. To sum up, measurements of a-RNA Pol III antibodies are useful in routine clinical practice because they are one of the marker antibodies in SSc diagnosis and in many cases they identify the subset of the disease with severe skin and renal involvement. Furthermore, the presence of a-RNA Pol III antibodies should alert physicians to an increased risk of scleroderma renal crisis and monitoring of blood pressure for early detection of this complication since the prompt introduction of angiotensin converting enzyme inhibitor can be life-saving. Moreover, the presence of a-RNA Pol III antibodies in SSc patients requires screening for associated cancer, even in the absence of clinical signs.

\section{Conflict of interest}

The authors declare no conflict of interest. 


\section{References}

1. Jeong S, Hwang H, Roh J, et al. Evaluation of an automated screening assay, compared to indirect immunofluorescence, an extractable nuclear antigen assay, and a line immunoassay in a large cohort of asian patients with antinuclear antibody-associated rheumatoid diseases: a multicenter retrospective study. J Immunol Res 2018; 2018: doi: 10.1155/2018/9094217

2. van den Hoogen F, Khanna D, Fransen J, et al. 2013 classification criteria for systemic sclerosis: an American College of Rheumatology/European League Against Rheumatism collaborative initiative. Ann Rheum Dis 2013; 72: 1747-55.

3. Kuwana M. Circulating anti-nuclear antibodies in systemic sclerosis: utility in diagnosis and disease subsetting. J Nippon Med Sch 2017; 84: 56-63.

4. Satiago M, Baron M, Burlingame RW, et al. Antibodies to RNA polymerase III in systemic sclerosis as detected by an Elisa. J Rheumatol 2007; 34: 1528-34.

5. Meyer O, De Chaisemartin L, Nicaise R, et al. Anti-RNA polymerase III antibody prevalence and associated clinical manifestations in a large series of French patients with systemic sclerosis: a-cross-sectional study. J Rheumatol 2010; 37: 125-30.

6. Terras S, Hartenstein H, Höxtermann S, et al. RNA polymerase III autoantibodies may indicate renal and more severe skin involvement in systemic sclerosis. Int J Dermatol 2016; 55: 882-5.

7. Hoffmann-Vold AM, Midtvedt $\varnothing$, Tennøe AH, et al. Cardiopulmonary disease development in anti-RNA polymerase IIIpositive systemic sclerosis: comparative analyses from an unselected, prospective patient cohort. J Rheumatol 2017; 44: 459-65.

8. Motegi S, Toki S, YamadaK, et al. Demographic and clinical features of systemic sclerosis patients with anti-RNA polymerase III antibodies. J Dermatol 2015; 42: 189-92.

9. Moinzadeh P, Fonseca C, Hellmich M, et al. Association of anti-RNA polymerase III autoantibodies and cancer in scleroderma. Arthritis Res Ther 2014; 16: R53.

10. Lazzaroni MG, Cavazzana I, Colombo E, et al. Malignancies in patients with anti-RNA polymerase III antibodies and systemic sclerosis: analysis of the EULAR scleroderma trials and research cohort and possible recommendations for screening. J Rheumatol 2017; 44: 639-47.

11. LeRoy EC, Black C, Fleishmajer R Scleroderma (systemic sclerosis): classification, subsets and pathogenesis. J Rheumatol 1988; 15: 202-4

12. Patterson KA, Roberts-Thomson PJ, Lester S, et al. interpretation of an extended autoantibody profile in a well-characterized Australian systemic sclerosis (scleroderma) cohort using principal components analysis. Arthritis Rheumatol 2015; 67: 3234-44.

13. Mierau R, Moinzadeh P, Riemekasten G, et al. Frequency of disease-associated and other nuclear autoantibodies in patients of the German Network for Systemic Scleroderma: correlation with characteristic clinical features. Arthritis Res Ther 2011; 13: R172.

14. Michalska-Jakubus M, Kowal M, Adamczyk M, Krasowska D. Anti-endothelial cell antibodies do not correlate with disease activity in systemic sclerosis. Adv Dermatol Allergol 2018; 35: 185-91.

15. Satoh T, Ishikawa O, Ihn H, et al. Clinical usefulness of antiRNA polymerase III antibody measurement by enzymelinked immunosorbent assay. Rheumatology 2009; 48: 1570-4.
16. Liaskos C, Marou E, Simopoulou T, et al. Disease-related autoantibody profile in patients with systemic sclerosis. Autoimmunity 2017; 50: 414-21.

17. Wielosz E, Dryglewska M, Majdan M. Serological profile of patients with systemic sclerosis. Postepy Hig Med Dosw 2014; 68: 987-91.

18. Sobanski V, Dauchet L, Lefèvre G, et al. Prevalence of antiRNA polymerase III antibodies in systemic sclerosis: new data from a French cohort and a systematic review and meta-analysis. Arthritis Rheumatol 2014; 66: 407-17.

19. Iniesta Arandia N, Simeón-Aznar CP, Guillén Del Castillo A, et al. Influence of antibody profile in clinical features and prognosis in a cohort of Spanish patients with systemic sclerosis. Clin Exp Rheumatol 2017; 106: 98-105.

20. Gordon SM, Stitt RS, Nee R, et al. Risk factors for future scleroderma renal crisis at systemic sclerosis diagnosis. J Rheumatol 2019; 46: 85-92.

21. Monfort JB, Mathian A, Amoura Z, et al. Cancers associated with systemic sclerosis involving anti-RNA polymerase III antibodies. Ann Dermatol Venereol 2018; 145: 33-6.

22. Saigusa R, Asano Y, Nakamura K, et al. Association of antiRNA polymerase III antibody and malignancy in Japanese patients with systemic sclerosis. J Dermatol 2015; 42: 524-7.

23. Bernal-Bello D, de Tena JG, Guillén-Del Castillo A, et al. Novel risk factors related to cancer in scleroderma. Autoimmun Rev 2017; 16: 461-8.

24. Lescoat A, Ballerie A, Belhomme N, et al. Synovial involvement assessed by power Doppler ultra-sonography in systemic sclerosis: results of a cross-sectional study. Rheumatology 2018; 57: 2012-21. 